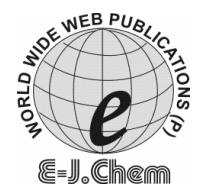

http://www.e-journals.net
ISSN: 0973-4945; CODEN ECJHAO

E-Journal of Chemistry 2009, 6(3), 928-937

\title{
Synthesis and Characterization of New Heterocyclic Liquid Crystals
}

\author{
D. SRIVIDHYA*, S. MANJUNATHAN and S. THIRUMARAN ${ }^{\S}$ \\ Department of Chemistry, Sona College of Technology, \\ Salem - 636005, Tamilnadu, India. \\ ${ }^{\S}$ Department of Chemistry, Annamalai University, \\ Annamalainagar - 608002, Tamilnadu, India. \\ srividhyad2000@yahoo.co.in
}

Received 27 March 2009; Accepted 30 April 2009

\begin{abstract}
This investigation enumerates the synthesis and mesomorphic properties of 1,2,3-triazole containing azobenzene liquid crystals. In these liquid crystals the methylene chain length at non polar end was varied from six to ten carbons to investigate the association properties of non polar chain on the melt. The compound was designed to have a polar ether chain at the other side of the molecule adjacent to the triazole ring and synthesized to enhance the dipolar interactions. These alterations in chemical structure produce two series of new liquid crystalline compounds with each series containing five variations in the methylene chain. The structure of the target compounds and the intermediates were confirmed by the ${ }^{1} \mathrm{H}$ NMR, ${ }^{13} \mathrm{C}$ NMR and IR spectral techniques. Polarized microscopic studies revealed that all the compounds in the series exhibited enantiotropic liquid crystalline properties. This was further confirmed using differential scanning calorimetric experiments. The energy minimized structure supports the mesogenic behavior of the structure.
\end{abstract}

Keywords: Triazole, Liquid crystals, Mesomophism, Azide alkyne cycloaddition.

\section{Introduction}

The design of novel thermotropic liquid crystals as advanced functional materials, involves suitable selection of core fragments, linking groups and terminal functionalities ${ }^{1}$. The preparation of new types of liquid crystal materials with rings other than six membered rings has received considerable attention because of their non-linearity and less mesogenic stability $^{2-4}$. Particularly, liquid crystalline compounds containing five-membered heterocyclic rings have been successfully evaluated ${ }^{5}$ and in particular, 1,2,3-triazoles are 
useful targets in chemical synthesis because of their high levels of biological activity ${ }^{6}$ and there by exhibiting great potential usage in material chemistry ${ }^{7}$. In addition, the presence of the heteroatom has lead to the increase in molecular dipole and dielectric anisotropy, thereby producing the molecular level polar organisations through generating electrostatic and molecular shape dependent interactions between molecules ${ }^{8}$. The compounds containing five membered 1,2,3- triazole ring, formed by the copper catalysed [2+3] dipolar cycloaddition reaction of organic azides and terminal alkynes, also known as 'click reaction', has attracted recent attention owing to the following advantageous properties: (i) a high chemical stability (in general, being inert to severe hydrolytic, oxidizing and reducing conditions, even at high temperature), (ii) a strong dipole moment (5.2-5.6 D), (iii) an aromatic character and (iv) a good hydrogen-bond-accepting ability ${ }^{9,10}$. In the field of mesogenic materials, only a very few liquid crystals has been synthesized containing 1,2,3-triazole ring ${ }^{11,12}$. Among those compounds, interestingly 1,2,3-triazole ring placed at a terminal position of a calametic mesogen linked with non-polar alkyl chains were not showing any liquid crystalline properties $^{13-15}$. We have synthesized two homologous series of non-linear heterocyclic azobenzene based liquid crystals, using $\mathrm{Cu}(\mathrm{I})$ catalysed 1,3-dipolar cycloaddition reaction and have investigated the mesomorphic properties.

\section{Experimental}

\section{Materials and methods}

Silica gel (MN Kieselgel 60-120 mesh) was used for column chromatography. The organic solvents diethyl ether, dichloromethane, benzene, methanol, ethanol and acetone were purified as per reported procedure ${ }^{16}$. Palladium on charcoal(10\%), ammonium chloride, acetic acid, 1-hexanol, 1-heptanol, 1-octanol, 1-nonanol, 1-decanol, potassium iodide, $\mathrm{HBr}$ 47\% (SRL), sodium hydroxide, potassium carbonate, sodium ascorbate, copper sulphate pentahydrate (SD fine \& E-Merck, India) were used without purification. 4-Azidophenol was prepared from 4-aminophenol by diazo-transfer reaction using the azide nucleophile as per the reported procedure ${ }^{17}$. The alkyl propargyl ethers were prepared by the reaction of propargyl alchocol and methyl/ethyl sulphate ${ }^{18}$.

The chemical structure of the intermediates and the target materials were analyzed by infrared spectroscopy with a Bruker IFS 66V Fourier Transform spectrophotometer using $\mathrm{KBr}$ pellets and nuclear magnetic resonance spectroscopy using Joel EX-400 FTNMR spectrometer in $\mathrm{CDCl}_{3}$ with TMS as an internal standard. Thin layer chromatography was performed with TLC sheets coated with silica; spots were detected by UV irradiation. Transition temperatures and phase transition enthalpies were determined by Differential Scanning Calorimetry (DSC) using a Perkin-Elmer DSC7 calorimeter at a heating rate of $5{ }^{\circ} \mathrm{C} \mathrm{min}-1$. The liquid crystalline texture of all the compounds were studied by using a Euromax Polarizing Microscope equipped with a Linkem HFS91 heating stage and a TP93 temperature programmer. The samples were made by placing a small quantity of the material between two thin glass cover slips, and the anisotropic behavior observed by heating and/or cooling at the rates of $5{ }^{\circ} \mathrm{C} \mathrm{min}^{-1}$. The photographs were taken with a Nikon FM10 camera and exposed on a Konica film.

\section{Synthesis of 4-(methoxymethyl)-1-(4-nitrophenyl)-1H-1,2,3-triazole (3a)}

3-Methoxy prop-1-yne (1a) (0.1 mole, $7.0 \mathrm{~g})$ and 4-azido nitrophenol (2) (0.1 mole, $16.4 \mathrm{~g})$ were dissolved in tert-butyl alcohol $(20 \mathrm{~mL})$. Sodium ascorbate $(10$ mole $\%)$ and copper sulphate pentahydrate $(10$ mole $\%)$ were dissolved in $2 \mathrm{~mL}$ of water and then added to the 
mixture at room temperature. Immediately, the color of the solution changed to orange and it was further stirred for $24 \mathrm{~h}$ till the colour changed to yellowish green. The solvent was removed under reduced pressure and the residue was purified by column chromatography on silica gel using hexane / ethyl acetate (4:1) as the eluent to give pure triazoles. Similar procedure was adopted for the preparation of 4-(ethoxymethyl)-1-(4-nitrophenyl)-1H-1,2,3-triazole.

${ }^{1} \mathrm{H}-\mathrm{NMR}\left(\mathrm{CDCl}_{3}, \delta \mathrm{ppm}\right): 3.47$ (s, 3H, O-C $\left.\underline{\mathrm{H}}_{3}\right), 4.67$ (s, 2H, Ar-O-C $\left.\underline{H}_{2}\right), 8.10(\mathrm{~s}, 1 \mathrm{H}$, Triazole ring C-H $), 7.96(\mathrm{~d}, 1 \mathrm{H}, \mathrm{Ar}-\mathrm{H}), 8.42(\mathrm{~d}, 1 \mathrm{H}, \mathrm{Ar}-\mathrm{H}) ;{ }^{13} \mathrm{C}-\mathrm{NMR}\left(\mathrm{CDCl}_{3}, \delta \mathrm{ppm}\right)$ : 58.69, 65.88, 147.23, 120.47, 141.16, 120.36, 125.57, 146.97 .

\section{4-(Ethoxymethyl)-1-(4-nitrophenyl)-1H-1,2,3-triazole (3b)}

${ }^{1} \mathrm{H}-\mathrm{NMR}\left(\mathrm{CDCl}_{3}, \delta \mathrm{ppm}\right): 1.28$ (t, 3H, O-CH$\left.{ }_{2}-\underline{\mathrm{H}}_{3}\right), 3.67$ (q, $\left.2 \mathrm{H}, \mathrm{O}-\underline{\mathrm{H}}_{2}\right), 4.74$ (s, $2 \mathrm{H}$, Ar-O-C $\left.\underline{H}_{2}\right), 8.14$ (s, $1 \mathrm{H}$, Triazole ring $\left.\mathrm{C}-\underline{\mathrm{H}}\right), 8.00$ (d, $\left.1 \mathrm{H}, \mathrm{Ar}-\mathrm{H}\right), 8.42(\mathrm{~d}, 1 \mathrm{H}, \mathrm{Ar}-\mathrm{H})$; ${ }^{13} \mathrm{C}-\mathrm{NMR}\left(\mathrm{CDCl}_{3}, \delta \mathrm{ppm}\right)$ : 18.69, 58.64, 65.88, 147.39, 120.47, 141.86, 120.26, 124.59, 146.97.

\section{Synthesis of \{4-[4-(methoxymethyl)-1H-1,2,3-triazol-1-1]phenyl\} amine (4a)}

4-(Methoxymethyl)-1-(4-nitrophenyl)-1H-1,2,3-triazole (3a) (0.05 mole, 11.7 g) was dissolved in dry ethanol, palladium on charcoal (10 wt $\%)$ was added and stirred under hydrogen atmosphere ( $1 \mathrm{~atm})$. The reaction was followed by thin layer chromatography. The solution was filtered through celite bed after the reaction was completed. The solvent was removed under reduced pressure. The residue obtained was chromatographed over silica gel using chloroform as solvent. The similar procedure was adopted for the preparation of \{4-[4(ethoxymethyl)-1H-1,2,3-triazol-1-1] phenyl $\}$ amine.

Synthesis of 4-\{[4-(-4-(methoxymethyl)-1H-1,2,3-triazol-1-1)phenyl)-diazenyl]phenol\} (5a)

To a solution of \{4-[4-(methoxymethyl)-1H-1,2,3-triazol-1-yl]phenyl $\}$ amine (4a) $(0.02$ mole, $4.1 \mathrm{~g})$ in conc. hydrochloric acid $(15 \mathrm{~mL})$, a solution of sodium nitrite $(0.04$ mole, $2.76 \mathrm{~g})$ in water $(5 \mathrm{~mL})$ was added drop wise at $0-5{ }^{\circ} \mathrm{C}$. The addition was adjusted in such a way that the temperature of the reaction mixture did not exceed $8{ }^{\circ} \mathrm{C}$. Phenol $(0.03$ mole, $2.76 \mathrm{~g})$ in dilute $\mathrm{NaOH}$ solution was added. The solution was stirred at $5-8{ }^{\circ} \mathrm{C}$ for one hour and finally, cold water $(200 \mathrm{~mL})$ was added slowly with stirring. The waxy precipitate formed was extracted using ether and dried over anhydrous magnesium sulphate. The solvent was removed using rotary vacuum evaporator and the residue obtained was purified using column chromatography. Similar procedure was adopted for the preparation of $4-\{[4-(-4-($ Ethoxymethyl $)-1 \mathrm{H}-$ 1,2,3-triazol-1-1)phenyl)-diazenyl]-phenol\}.

${ }^{1} \mathrm{H}-\mathrm{NMR}\left(\mathrm{CDCl}_{3}, \delta \mathrm{ppm}\right): 3.63$ (s, 3H, O-C $\left.\underline{\mathrm{H}}_{3}\right), 4.75$ (s, 2H, Ar-O-C $\left.\underline{\mathrm{H}}_{2}\right), 8.02$ (s, 1H, triazole ring $\mathrm{C}-\underline{\mathrm{H}}), 7.02(\mathrm{~d}, 1 \mathrm{H}, \mathrm{Ar}-\mathrm{H}), 7.88(\mathrm{~d}, 1 \mathrm{H}, \mathrm{Ar}-\mathrm{H}), 7.94(\mathrm{~d}, 1 \mathrm{H}, \mathrm{Ar}-\mathrm{H}), 8.05$ (d, 1H, Ar-H); ${ }^{13} \mathrm{C}-\mathrm{NMR}\left(\mathrm{CDCl}_{3}, \mathrm{ppm}\right): 57.96,65.32,151.95,121.21,137.99,122.71,124.03,145.69$, $125.61,116.52,161.85$.

4-\{[4-(-4-(Ethoxymethyl)-1H-1,2,3-triazol-1-1)phenyl)-diazenyl]-phenol\} (5b)

${ }^{1} \mathrm{H}-\mathrm{NMR}\left(\mathrm{CDCl}_{3}, \delta \mathrm{ppm}\right): 1.22$ (t, 3H, O-CH$\left.{ }_{2} \mathrm{CH}_{3}\right), 3.61$ (q, 2H, O-C $\left.\underline{\mathrm{H}}_{2}\right), 4.67$ (s, 2H, Ar-O$\left.\mathrm{CH}_{2}\right), 7.98(\mathrm{~s}, 1 \mathrm{H}$, Triazole ring $\mathrm{C}-\underline{\mathrm{H}}), 6.92(\mathrm{~d}, 1 \overline{\mathrm{H}}, \mathrm{Ar}-\mathrm{H}), 7.81(\mathrm{~d}, 1 \overline{\mathrm{H}}, \mathrm{Ar}-\mathrm{H}), 7.95(\mathrm{~d}, 1 \mathrm{H}$, $\mathrm{Ar}-\mathrm{H}), 7.85$ (d, $1 \mathrm{H}, \mathrm{Ar}-\mathrm{H}) ;{ }^{13} \mathrm{C}-\mathrm{NMR}\left(\mathrm{CDCl}_{3}, \delta \mathrm{ppm}\right): 19.81,57.36,64.92,152.05,121.51$, $138.99,122.71,124.03,146.59,126.61,117.52,161.55$. 
General procedure for the preparation of 4-\{(4-[4-(alkoxymethyl)-1H-1,2,3-triazol1-1]phenyl)-diazenyl\}-1-(alkyloxy)benzene (Ia-e and IIa-e)

4-\{[4-(-4-(Alkoxymethyl)-1H-1,2,3-triazol-1-1)phenyl)-diazenyl]phenol\} (5a) (0.004 mole, $1.4 \mathrm{~g})$ was dissolved in dry acetone to which finely powdered anhydrous potassium carbonate $(0.04$ mole, $5.4 \mathrm{~g})$ was added and simultaneously stirred under reflux condition. Catalytic amount of potassium iodide (10 wt \%) was added to it and $n$-bromoalkane (6a-e) $(0.005$ mole, $1.2 \mathrm{~mL})$ dissolved in acetone was added to the refluxing solution over a period of $1 \mathrm{~h}$ and the heating was continued for another $48 \mathrm{~h}$ and the reaction was followed by TLC. Once the reaction was completed, the reaction mixture was filtered and washed with acetone. Washings were added to the main fraction and evaporated. The solid obtained was purified by column chromatography over silica gel using chloroform as a solvent.

4-\{(4-[4-(Methoxymethyl)-1H-1,2,3-triazol-1-1]phenyl)-diazenyl\}-1-(hexyloxy) benzene (Ia)

IR $\left(\mathrm{KBr}, \mathrm{cm}^{-1}\right): 1254$ (C-O-C), 3138 (triazole C-H), 1603 (Ar C-C), 1473 (N=N Unsymm), 1044 (Aryl-alkyl ether), 2935 (Aliphatic C-H); ${ }^{1} \mathrm{H}-\mathrm{NMR}\left(\mathrm{CDCl}_{3}, \delta \mathrm{ppm}\right): 3.48\left(\mathrm{~s}, 3 \mathrm{H},-\mathrm{CH}_{2}-\right.$ $\left.\mathrm{O}-\mathrm{CH}_{3}\right), 4.68$ (s, 2H,Ar-O-C $\left.\underline{H}_{2}\right), 8.04$ (s, 1H, Triazole ring $\left.\mathrm{C}-\underline{\mathrm{H}}\right), 7.93(\mathrm{~d}, 1 \mathrm{H}, \mathrm{Ar}-\mathrm{H}), 8.01$ $(\mathrm{d}, 1 \mathrm{H}, \mathrm{Ar}-\mathrm{H}), 7.87(\mathrm{~d}, 1 \mathrm{H}, \mathrm{Ar}-\mathrm{H}), 7.01(\mathrm{~d}, 1 \mathrm{H}, \mathrm{Ar}-\mathrm{H}), 4.04$ (t, $\left.2 \mathrm{H}, \mathrm{Ar}-\mathrm{O}-\mathrm{CH}_{2}\right), 0.80-1.90$ $(\mathrm{m}, 11 \mathrm{H}, \mathrm{Al}-\mathrm{H}) ;{ }^{13} \mathrm{C}-\mathrm{NMR}\left(\mathrm{CDCl}_{3}, \delta \mathrm{ppm}\right): 58.51,64.09,152.35,120.47,137.79,120.83$, $125.07,146.47,146.69,123.96,114.79,162.21,68.43,14.12-31.89$.

4-\{(4-[4-(Methoxymethyl)-1H-1,2,3-triazol-1-1]phenyl)-diazenyl\}-1-(heptyloxy)benzene (Ib) IR $\left(\mathrm{KBr}, \mathrm{cm}^{-1}\right): 1253$ (C-O-C), 3129 (triazole C-H), 1602 (Ar C-C), 1469 (N=N Unsymm), 1044 (Aryl-alkyl ether), 2921 (Aliphatic C-H); ${ }^{1} \mathrm{H}-\mathrm{NMR}\left(\mathrm{CDCl}_{3}, \delta \mathrm{ppm}\right): 3.67$ (s, 3H, - $\mathrm{CH}_{2^{-}}$ $\left.\mathrm{O}-\mathrm{C}_{3}\right), 4.73$ (s, 2H, Ar-O-C $\left.\underline{\mathrm{H}}_{2}\right), 7.99$ (s, 1H, Triazole ring C- $\left.\underline{\mathrm{H}}\right), 7.79$ (d, 1H, Ar-H), 8.15 $(\mathrm{d}, 1 \mathrm{H}, \mathrm{Ar}-\mathrm{H}), 7.38(\mathrm{~d}, 1 \mathrm{H}, \mathrm{Ar}-\mathrm{H}), 6.99(\mathrm{~d}, 1 \mathrm{H}, \mathrm{Ar}-\mathrm{H}), 4.05\left(\mathrm{t}, 2 \mathrm{H}, \mathrm{Ar}-\mathrm{O}-\mathrm{C}_{2}\right), 0.87-1.86$ $(\mathrm{m}, 13 \mathrm{H}, \mathrm{Al}-\mathrm{H}) ;{ }^{13} \mathrm{C}-\mathrm{NMR}\left(\mathrm{CDCl}_{3}, \delta \mathrm{ppm}\right): 64.09,66.31,152.35,120.47,137.83,120.84$, 125.06, 146.48, 146.70, 123.95, 114.80, 162.21, 68.43, 14.08-31.76.

4-\{(4-[4-(Methoxymethyl)-1H-1,2,3-triazol-1-1]phenyl)-diazenyl\}-1-(octyloxy)benzene (Ic) IR (KBr, cm $\left.{ }^{-1}\right): 1253$ (C-O-C), 3129 (triazole C-H), 1605 (Ar C-C), 1470 (N=N Unsymm), 1045 (Aryl-alkyl ether), 2922 (Aliphatic C-H); ${ }^{1} \mathrm{H}-\mathrm{NMR}\left(\mathrm{CDCl}_{3}, \delta \mathrm{ppm}\right): 3.63$ (s, 3H, - $\mathrm{CH}_{2^{-}}$ $\left.\mathrm{O}-\mathrm{CH}_{3}\right), 4.74$ (s, 2H, Ar-O-C $\left.\underline{H}_{2}\right), 8.06$ (s, 1H, Triazole ring $\left.\mathrm{C}-\underline{\mathrm{H}}\right), 7.94(\mathrm{~d}, 1 \mathrm{H}, \mathrm{Ar}-\mathrm{H}), 8.03$ (d, 1H, Ar-H), 7.88 (d, 1H, Ar-H), 7.02 (d, 1H, Ar-H), 4.05 (t, 2H, Ar-O-C $\left.\underline{H}_{2}\right), 0.90-1.85$ (m, 15H, Al-H); ${ }^{13} \mathrm{C}-\mathrm{NMR}\left(\mathrm{CDCl}_{3}, \delta \mathrm{ppm}\right): 62.66,64.87,150.92,119.02,136.40,119.42$, 123.61, 145.05, 145.27, 122.51, 113.36, 160.76, 66.99, 12.57-30.11.

\section{4-\{(4-[4-(Methoxymethyl)-1H-1,2,3-triazol-1-1]phenyl)-diazenyl\}-1-(nanoyloxy)benzene (Id)}

IR (KBr, cm $\left.{ }^{-1}\right)$ : 1252 (C-O-C), 3129 (triazole C-H), 1602 (Ar C-C), 1468 (N=N Unsymm), 1041 (Aryl-alkyl ether), 2929 (Aliphatic C-H); ${ }^{1} \mathrm{H}-\mathrm{NMR}\left(\mathrm{CDCl}_{3}, \delta \mathrm{ppm}\right): 3.65$ (s, 3H, - $\mathrm{CH}_{2^{-}}$ $\left.\mathrm{O}-\mathrm{C}_{3}\right), 4.75\left(\mathrm{~s}, 2 \mathrm{H}, \mathrm{Ar}-\mathrm{O}-\mathrm{C}_{2}\right), 8.05(\mathrm{~s}, 1 \mathrm{H}$, Triazole ring $\mathrm{C}-\underline{\mathrm{H}}), 7.90(\mathrm{~d}, 1 \mathrm{H}, \mathrm{Ar}-\mathrm{H}), 8.00$ (d, $1 \mathrm{H}, \mathrm{Ar}-\mathrm{H}), 7.85$ (d, 1H, Ar-H), 7.00 (d, 1H, Ar-H), 4.05 (t, 2H, Ar-O-C $\left.\underline{\mathrm{H}}_{2}\right), 0.90-1.85$ $(\mathrm{m}, 17 \mathrm{H}, \mathrm{Al}-\mathrm{H}) ;{ }^{13} \mathrm{C}-\mathrm{NMR}\left(\mathrm{CDCl}_{3}, \delta \mathrm{ppm}\right): 64.10,66.31,152.34,120.47,137.84,120.84$, 125.07, 146.48, 146.71, 123.96, 114.80, 162.21, 68.44, 14.12-31.90.

\section{4-\{(4-[4-(Methoxymethyl)-1H-1,2,3-triazol-1-1]phenyl)-diazenyl\}-1-(decyloxy)benzene (Ie)}

IR $\left(\mathrm{KBr}, \mathrm{cm}^{-1}\right): 1255$ (C-O-C), 3123 (triazole C-H), 1603 (Ar C-C), 1473 (N=N Unsymm), 1045 (Aryl-alkyl ether), 2919 (Aliphatic C-H); ${ }^{1} \mathrm{H}-\mathrm{NMR}\left(\mathrm{CDCl}_{3}, \delta \mathrm{ppm}\right): 3.67$ (s, 3H, - $\mathrm{CH}_{2}-$ $\left.\mathrm{O}-\mathrm{CH}_{3}\right), 4.72\left(\mathrm{~s}, 2 \mathrm{H}, \mathrm{Ar}-\mathrm{O}-\mathrm{CH}_{2}\right), 8.05(\mathrm{~s}, 1 \mathrm{H}$, Triazole ring $\mathrm{C}-\underline{\mathrm{H}}), 7.93(\mathrm{~d}, 1 \mathrm{H}, \mathrm{Ar}-\mathrm{H}), 8.02$ (d, 1H, Ar-H), 7.88 (d, 1H, Ar-H), 7.01 (d, 1H, Ar-H), 4.04 (t, 2H, Ar-O-C $\left.\underline{H}_{2}\right), 0.88-1.89$ 
(m, 19H, Al-H); ${ }^{13} \mathrm{C}-\mathrm{NMR}\left(\mathrm{CDCl}_{3}, \delta \mathrm{ppm}\right): 64.08,65.96,152.32,120.46,137.82,120.79$, 125.06, 146.12, 146.68, 123.95, 114.78, 162.20, 68.42, 14.12-31.89.

4-\{(4-[4-(Ethoxymethyl)-1H-1,2,3-triazol-1-1]phenyl)-diazenyl\}-1-(hexyloxy)benzene (IIa) IR $\left(\mathrm{KBr}, \mathrm{cm}^{-1}\right)$ : 1253 (C-O-C), 3128 (triazole C-H), 1603 (Ar C-C), 1469 (N=N Unsymm), 1045 (Aryl-alkyl ether), 2920 (Aliphatic C-H); ${ }^{1} \mathrm{H}-\mathrm{NMR}\left(\mathrm{CDCl}_{3}, \delta \mathrm{ppm}\right): 0.91$ (t, 3H, O-CH${ }_{2}-$ $\mathrm{CH} 3), 3.67$ (q, 2H, O-C $\left.\underline{\mathrm{H}}_{2}\right), 4.73$ (s, 2H, Ar-O-C $\left.\underline{\mathrm{H}}_{2}\right), 8.06$ (s, 1H, Triazole ring $\left.\mathrm{C}-\underline{\mathrm{H}}\right), 7.93$ (d, $1 \mathrm{H}, \mathrm{Ar}-\mathrm{H}), 8.02(\mathrm{~d}, 1 \mathrm{H}, \mathrm{Ar}-\mathrm{H}), 7.88(\mathrm{~d}, 1 \mathrm{H}, \mathrm{Ar}-\mathrm{H}), 7.01(\mathrm{~d}, 1 \mathrm{H}, \mathrm{Ar}-\mathrm{H}), 4.05$ (t, 2H, Ar-O$\left.\mathrm{CH}_{2}\right), 1.25-1.86(\mathrm{~m}, 1 \mathrm{H}, \mathrm{Al}-\mathrm{H}) ;{ }^{13} \mathrm{C}-\mathrm{NMR}\left(\mathrm{CDCl}_{3}, \delta \mathrm{ppm}\right): 15.16,68.39,64.09,151.10$, 120.72, 134.52, 120.90, 123.23, 146.33, 146.70, 123.39, 114.42, 163.82, 66.29, 14.11-31.53.

4-\{(4-[4-(Ethoxymethyl)-1H-1,2,3-triazol-1-1]phenyl)-diazenyl\}-1-(heptyloxy)benzene (IIb)

IR (KBr, cm $\left.{ }^{-1}\right): 1253$ (C - O - C), 3131 (triazole C-H), 1603 (Ar C-C), 1470 (N=N Unsymm), 1042 (Aryl-alkyl ether), 2934 (Aliphatic C-H); ${ }^{1} \mathrm{H}-\mathrm{NMR}\left(\mathrm{CDCl}_{3}, \delta \mathrm{ppm}\right): 0.90$ (t, 3H, O-CH${ }_{2}-$ $\left.\mathrm{CH}_{3}\right), 3.66$ (q, $\left.2 \mathrm{H}, \mathrm{O}-\mathrm{C}_{2}\right), 4.72$ (s, 2H, Ar-O-C $\left.\underline{H}_{2}\right), 7.99$ (s, 1H, Triazole ring $\left.\mathrm{C}-\underline{\mathrm{H}}\right), 7.79$ (d, $1 \mathrm{H}, \mathrm{Ar}-\mathrm{H}), 8.15(\mathrm{~d}, 1 \mathrm{H}, \mathrm{Ar}-\mathrm{H}), 7.38(\mathrm{~d}, 1 \mathrm{H}, \mathrm{Ar}-\mathrm{H}), 6.99(\mathrm{~d}, 1 \mathrm{H}, \mathrm{Ar}-\mathrm{H}), 4.05$ (t, 2H, Ar-O$\left.\mathrm{CH}_{2}\right), 1.26-1.86(\mathrm{~m}, 13 \mathrm{H}, \mathrm{Al}-\mathrm{H}) ;{ }^{13} \mathrm{C}-\mathrm{NMR}\left(\mathrm{CDCl}_{3}, \delta \mathrm{ppm}\right): 15.18,68.48,64.15,152.43$, $120.47,137.89,120.91,125.09,146.77,146.56,123.98,114.85,162.25,66.35,14.08-31.91$.

4-\{(4-[4-(Ethoxymethyl)-1H-1,2,3-triazol-1-1]phenyl)-diazenyl\}-1-(octtyloxy)benzene (IIc) IR (KBr, cm $\left.{ }^{-1}\right): 1229$ (C - O - C), 3138 (triazole C-H), 1602 (Ar C-C), 1473 (N=N Unsymm), 1041 (Aryl-alkyl ether), 2935 (Aliphatic C-H); ${ }^{1} \mathrm{H}-\mathrm{NMR}\left(\mathrm{CDCl}_{3}, \delta \mathrm{ppm}\right): 0.91$ (t, 3H, O-CH${ }_{2}-$ $\left.\mathrm{CH}_{3}\right), 3.67$ (q, $\left.2 \mathrm{H}, \mathrm{O}-\mathrm{C}_{2}\right), 4.74$ (s, $\left.2 \mathrm{H}, \mathrm{Ar}-\mathrm{O}-\underline{C}_{2}\right), 8.02$ (s, 1H, Triazole ring $\left.\mathrm{C}-\underline{\mathrm{H}}\right), 7.94$ (d, $1 \mathrm{H}, \mathrm{Ar}-\mathrm{H}), 8.05(\mathrm{~d}, 1 \mathrm{H}, \mathrm{Ar}-\mathrm{H}), 7.88(\mathrm{~d}, 1 \mathrm{H}, \mathrm{Ar}-\mathrm{H}), 7.02(\mathrm{~d}, 1 \mathrm{H}, \mathrm{Ar}-\mathrm{H}), 4.05$ (t, 2H, Ar-O-C $\left.\underline{\mathrm{H}}_{2}\right)$, $1.26-1.88(\mathrm{~m}, 15 \mathrm{H}, \mathrm{Al}-\mathrm{H}) ;{ }^{13} \mathrm{C}-\mathrm{NMR}\left(\mathrm{CDCl}_{3}, \delta \mathrm{ppm}\right): 15.18,68.48,64.14,152.43,120.47$, 137.89, 120.91, 125.09, 146.77, 146.55, 123.98, 114.85, 162.26, 66.35, 14.11-31.79.

\section{4-\{(4-[4-(Ethoxymethyl)-1H-1,2,3-triazol-1-1]phenyl)-diazenyl\}-1-(nanoyloxy)benzene (IId)}

IR $\left(\mathrm{KBr}, \mathrm{cm}^{-1}\right)$ : 1251 (C-O-C), 3129 (triazole C-H), 1602 (Ar C-C), 1469 (N=N Unsymm), 1044 (Aryl-alkyl ether), 2931 (Aliphatic C-H); ${ }^{1} \mathrm{H}-\mathrm{NMR}\left(\mathrm{CDCl}_{3}, \delta \mathrm{ppm}\right): 0.92$ (t, 3H, O-CH${ }^{-}$ $\left.\mathrm{CH}_{3}\right), 3.66$ (q, $\left.2 \mathrm{H}, \mathrm{O}-\mathrm{C}_{2}\right), 4.72$ (s, 2H, Ar-O-C $\left.\underline{H}_{2}\right), 7.99$ (s, 1H, Triazole ring $\left.\mathrm{C}-\underline{\mathrm{H}}\right), 7.78$ (d, $1 \mathrm{H}, \mathrm{Ar}-\mathrm{H}), 8.15(\mathrm{~d}, 1 \mathrm{H}, \mathrm{Ar}-\mathrm{H}), 7.38(\mathrm{~d}, 1 \mathrm{H}, \mathrm{Ar}-\mathrm{H}), 6.99$ (d, 1H, Ar-H), 4.05 (t, 2H, Ar-O$\left.\mathrm{C}_{2}\right), 1.26-1.86(\mathrm{~m}, 17 \mathrm{H}, \mathrm{Al}-\mathrm{H}) ;{ }^{13} \mathrm{C}-\mathrm{NMR}\left(\mathrm{CDCl}_{3}, \delta \mathrm{ppm}\right): 15.16,68.39,64.11,151.11$, $120.71,134.54,120.91,123.23,146.35,146.35,121.73,114.42,164.65,66.30,14.10-31.89$.

4-\{(4-[4-(Ethoxymethyl)-1H-1,2,3-triazol-1-1]phenyl)-diazenyl\}-1-(decyloxy)benzene (IIe)

IR $\left(\mathrm{KBr}, \mathrm{cm}^{-1}\right): 1255$ (C - O - C), 3128 (triazole C-H), 1604 (Ar C-C), 1470 (N=N Unsymm), 1045 (Aryl-alkyl ether), 2913 (Aliphatic C-H); ${ }^{1} \mathrm{H}-\mathrm{NMR}\left(\mathrm{CDCl}_{3}, \delta \mathrm{ppm}\right): 0.89$ (t, 3H, O- $\left.\mathrm{CH}_{2}-\underline{\mathrm{C}}_{3}\right), 3.67$ (q, 2H, O-C $\left.\underline{\mathrm{H}}_{2}\right), 4.74\left(\mathrm{~s}, 2 \mathrm{H}\right.$, Ar-O- $\left.\underline{\mathrm{H}}_{2}\right), 8.05$ (s, 1H, Triazole ring $\mathrm{C}-\underline{\mathrm{H}}), 7.94(\mathrm{~d}, 1 \mathrm{H}, \mathrm{Ar}-\mathrm{H}), 8.03(\mathrm{~d}, 1 \mathrm{H}, \mathrm{Ar}-\mathrm{H}), 7.88(\mathrm{~d}, 1 \mathrm{H}, \mathrm{Ar}-\mathrm{H}), 7.02(\mathrm{~d}, 1 \mathrm{H}, \mathrm{Ar}-\mathrm{H})$, $4.05\left(\mathrm{t}, 2 \mathrm{H}, \mathrm{Ar}-\mathrm{O}-\mathrm{C}_{2}\right), 1.26-1.85(\mathrm{~m}, 19 \mathrm{H}, \mathrm{Al}-\mathrm{H}) ;{ }^{13} \mathrm{C}-\mathrm{NMR}\left(\mathrm{CDCl}_{3}, \delta \mathrm{ppm}\right): 15.16$, $68.39,64.09,151.10,120.72,134.53,120.90,123.23,146.33,146.33,121.72,114.42$, 164.64, 66.29, 14.06-31.79.

\section{Results and Discussion}

The two series of nonlinear liquid crystalline compounds differing in their alkyl chain present in the terminal position of triazole ring were synthesized for the study of mesomorphic properties and their characteristics. 4-azido benzene (2) was coupled with alkyl propargyl ethers (methyl, ethyl ethers) to get the nitro substituted triazole compound (3). This was further reduced to get the amine compound (4). The amine was diazotized and 
reacted with phenol in basic condition to get the precursor compound (5). This was further alkylated using various alkyl bromides (6a-e) to get two series of compounds (Ia-e, IIa-e). The overall synthetic scheme is presented in Scheme-1. The compounds were characterized by various spectral techniques and the structures of the synthesized compounds were verified.

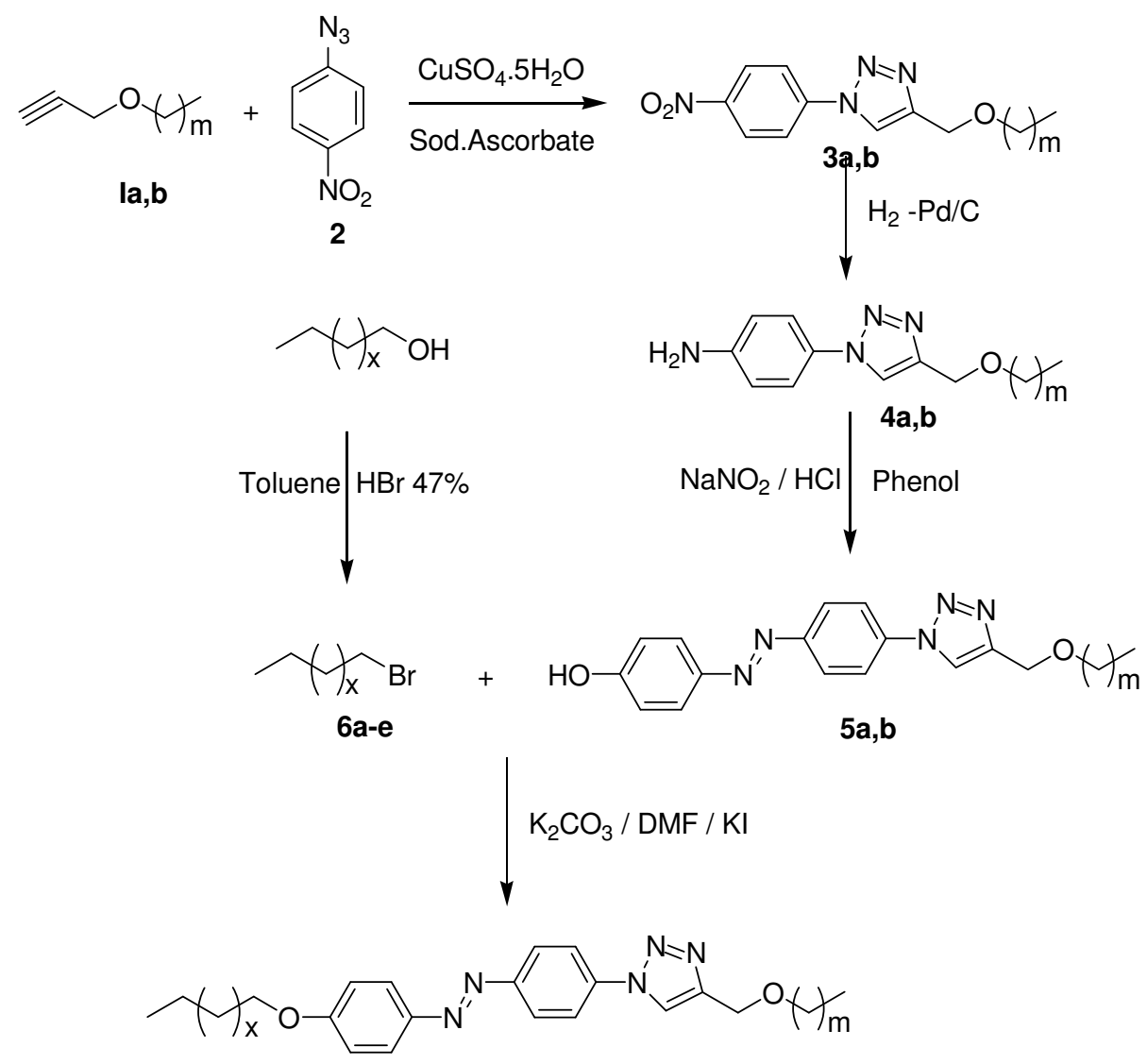

\begin{tabular}{|r|c|c|c|c|c|}
\hline $\mathrm{m} x$ & 3 & 4 & 5 & 6 & 7 \\
\hline 0 & la & Ib & Ic & Id & le \\
\hline 1 & Ila & Ilb & Ilc & Ild & Ile \\
\hline
\end{tabular}

Scheme 1. Synthesis of compounds.

Liquid crystalline behaviors of the compounds were investigated using hot stage polarizing microscopy. The compounds from both the series exhibits enantiotropic liquid crystalline properties. They were confirmed by repeated cooling and heating experiments with polarizing microscope. On slow cooling from the isotropic liquid, tiny batonnets developed at the edge of melted substance and grown up to the focal-conic fan texture, as shown in Figure 1, which is the characteristic of Sm A texture. Interestingly, the longer 
methylene chain compounds exhibit perfect focal domains and shorter methylene chain compounds show domain with strongly-reduced conics. The middle chains, exhibit half domain with one or more hyperbola branches. Thus all the compounds in I-II series are smectic with variety of domain structure depending upon their non-polar chain length.

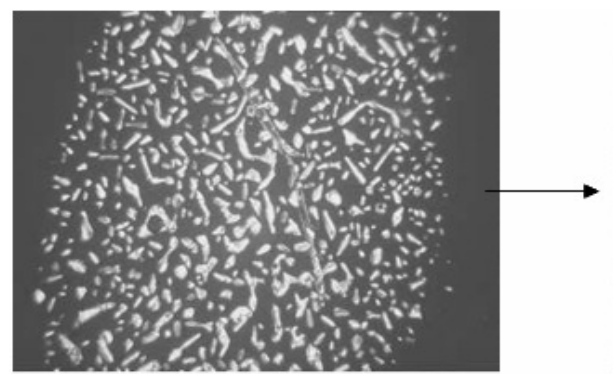

$192.8^{\circ} \mathrm{C}$

Smectic batonnets

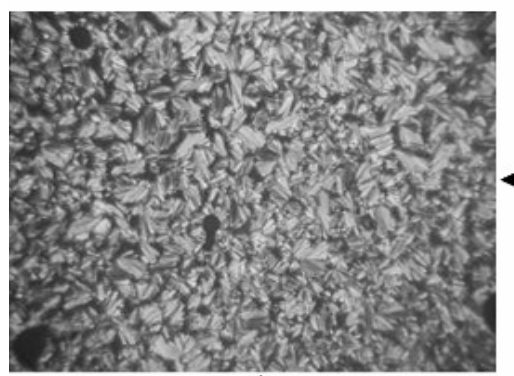

$178.3^{\circ} \mathrm{C}$

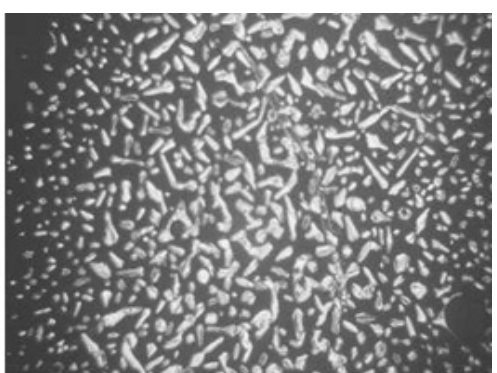

$191.2^{\circ} \mathrm{C}$

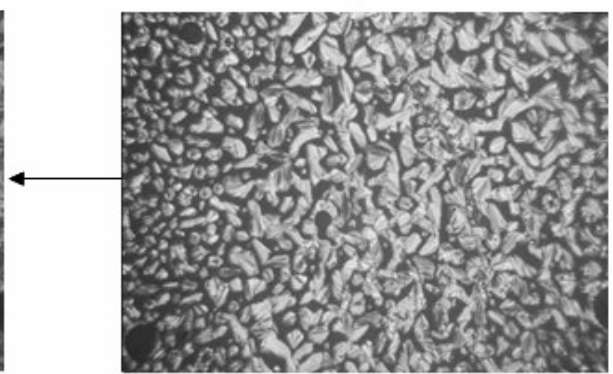

$188.8^{\circ} \mathrm{C}$

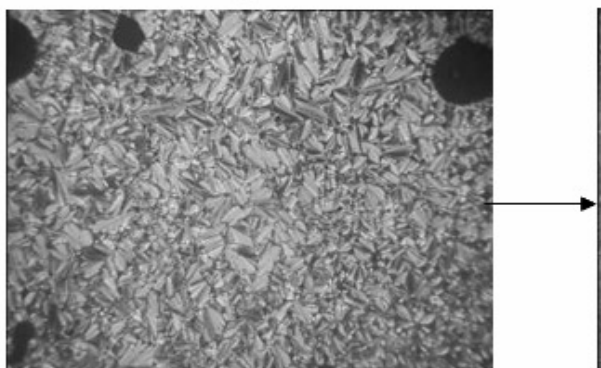

$120.0^{\circ} \mathrm{C}$

Smectic A mesophase

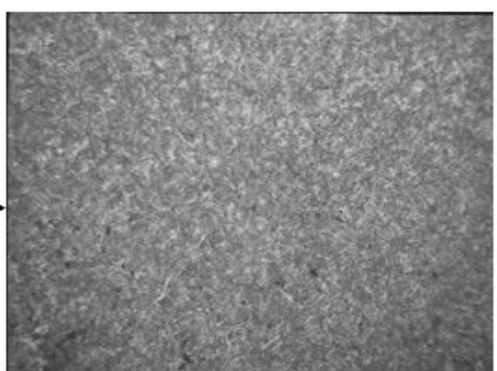

$113.7^{\circ} \mathrm{C}$

Crystalline

Figure 1. The representative HOPM photograph of IIb showing the mesophase formation while cooling from isotropic liquid-Smectic A-Crystalline thermal transitions.

For further confirmation, all the compounds were subjected to differential scanning calorimetric investigation, which showed two sharp endothermic peaks, corresponding to the melting temperature (Tm) (high enthalpy) and isotropic temperature (Ti) (low enthalpy) respectively, confirming the formation of liquid crystalline phase. Phase details and transition temperatures of all the compounds are summarized in Table 1. The DSC thermograms were shown in Figure 2. In general, by comparing all the transition temperatures, there is a decrease in the melting and isotropic transition temperatures while 
increasing the non-polar chain length. It may be due to the regular insertion of methylene groups that separate the mesogens, thereby adding flexibility apart from reducing the transition temperatures. The mesophase stability also shows the same trend. Though the mesogen was a non-linear, the polar ether group generates enough dipole in addition to triazole unit which helps to impart liquid crystalline properties.

(a)
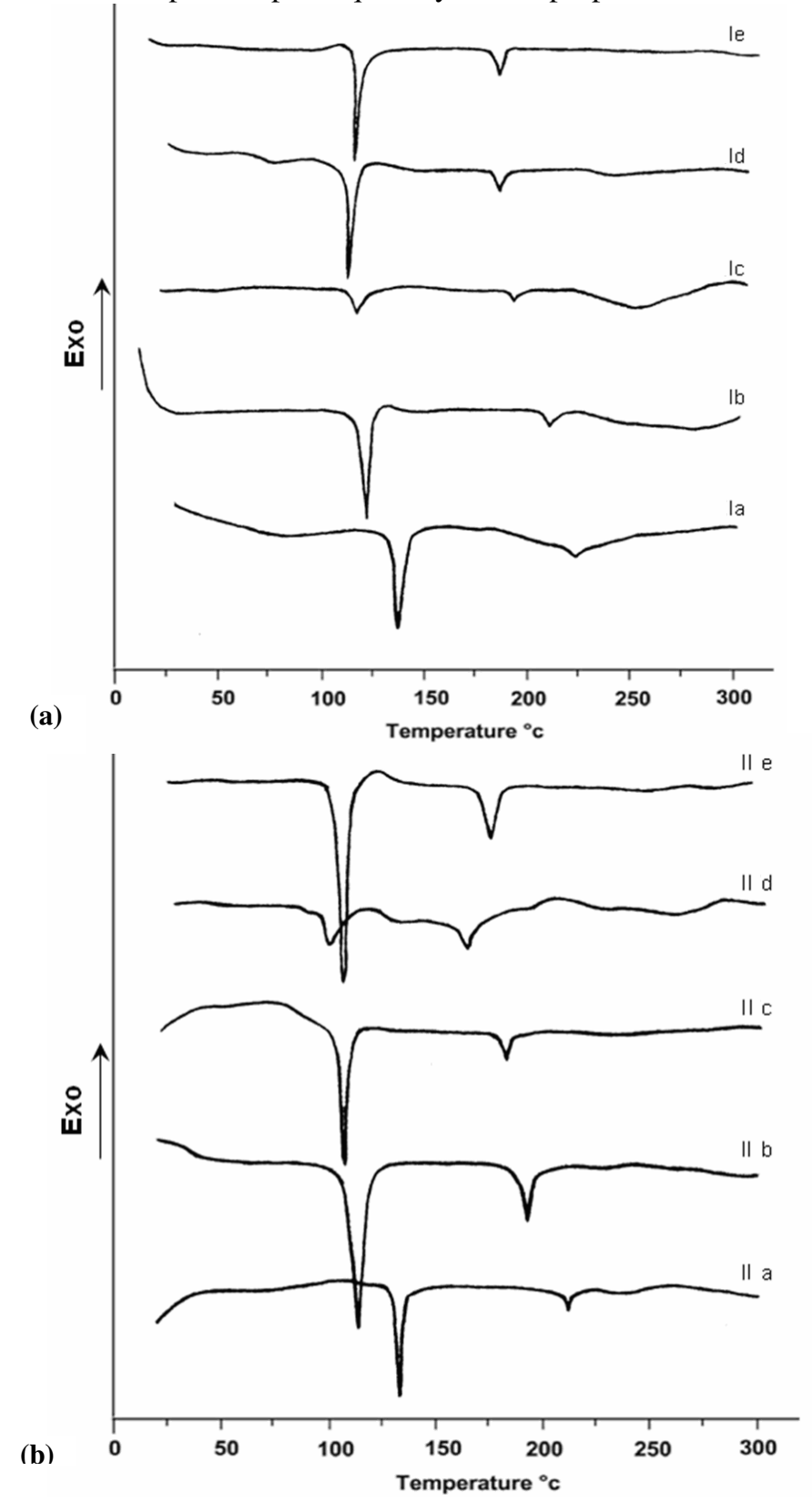

Figure 2.(a) DSC Thermograms of compounds Ia-e (b) DSC Thermograms of compounds IIa-e 
Table 1. Transition temperature of the compounds(I, II) observed in DSC.

\begin{tabular}{cccc}
\hline Compd. & $\mathrm{T}_{\mathrm{m}}(\mathrm{Cr}-\mathrm{Sm})$ & $\mathrm{T}_{\mathrm{i}}(\mathrm{Sm}-\mathrm{I})$ & $\Delta \mathrm{T}\left(\mathrm{T}_{\mathrm{i}}-\mathrm{T}_{\mathrm{m}}\right)$ \\
\hline Ia & 132 & 220 & 88 \\
Ib & 118 & 210 & 92 \\
Ic & 115 & 185 & 70 \\
Id & 109 & 175 & 66 \\
Ie & 110 & 175 & 65 \\
IIa & 130 & 213 & 83 \\
IIb & 115 & 193 & 78 \\
IIc & 110 & 182 & 72 \\
IId & 105 & 168 & 63 \\
IIe & 107 & 171 & 64 \\
\hline
\end{tabular}

Further, the rigidity of the mesogen was confirmed by using molecular modelling programme. The energy minimized structure of the model compound (Figure 3 ) which mimic the common structure of the mesogen of two series reveal that the triazole ring which is attached to the phenyl ring possess three electronegative nitrogen atoms and two carbon atoms tilted slightly along the axis passing through 1 and 4 carbons of phenyl ring (the line which bisects phenyl ring into two sections). From the bond angles, the deviation (bent angle) is calculated as $33^{\circ}$. The charge density in the entire carbon frame work is between 0.06 and 0.12 , but in the case of triazole nitrogen, the ipso nitrogen has minimum charge density as that of the carbon frame. The other two nitrogens in the ring showed elevated the charge density values of $-0.221 \&-0.313$, which increases the polarisation of the entire molecule along with the polarity of ether oxygen in the short chain attached to the triazole ring. This increased polarity assists the formation of smectic A mesophases in all these compounds.
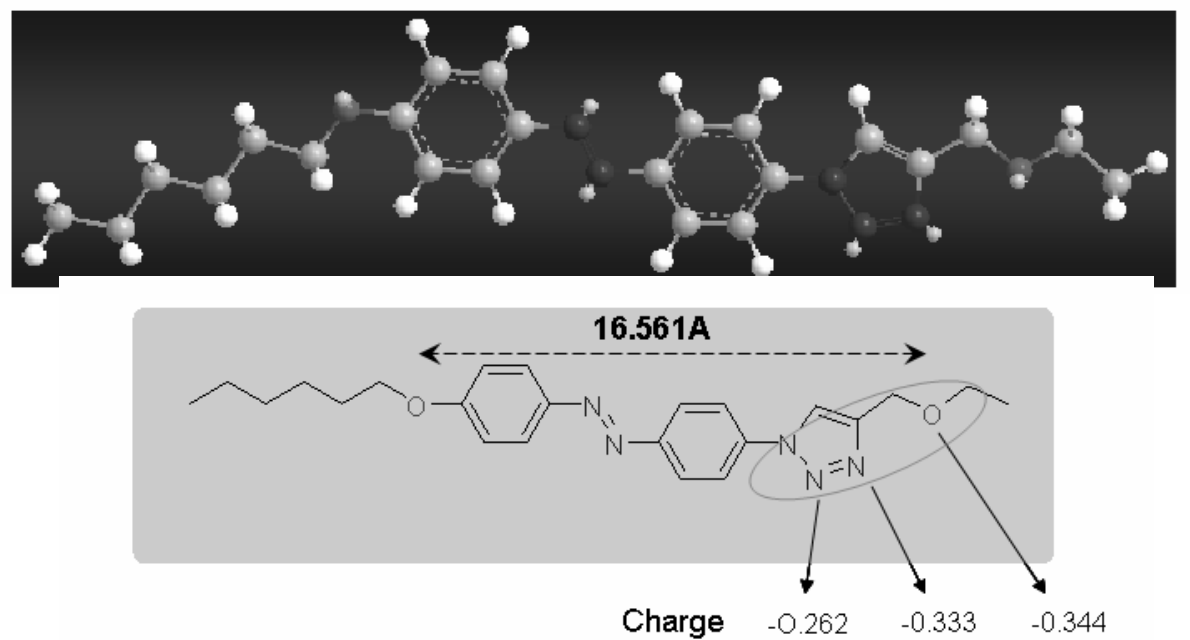

Figure 3. Energy minimized structure of the model compound

\section{Conclusion}

Two series of heterocyclic liquid crystalline compounds with the heterocyclic ring at the terminal position were synthesized and their mesomorphic properties were analyzed with 
respect to phase transition temperature and chemical structure. The structures of all the target compounds were confirmed by the ${ }^{1} \mathrm{H}$ NMR, ${ }^{13} \mathrm{C}$ NMR and IR spectroscopic methods. DSC measurements and HOPM investigation confirm the liquid crystalline nature of the synthesized compounds. Introduction of polar oxygen near to the heterocyclic ring produce liquid crystalline property, whereas it was absent in the case of non-polar alkyl chains attached directly to heterocyclic ring in these types of three ring compounds. The reason could be the dipole-dipole interaction of polar alkoxy groups which increases the attraction and leads to arrangement of molecule in the smectic layer structures.

\section{References}

1. Demus D, Goodby J, Gray G W, Spiess H W and Vill V, Handbook of liquid crystals, Wiely-VCH, New York, Vol 3, 1998.

2. Zaschke H, Wiss Z Univ., 1980, 3, 35.

3. Brown J W and Byron D J, Mol Cryst Liq Cryst., 1989, 173, 121.

4. Iglesias R, Serrano J L and Sierra T, Liq Cryst., 1997, 321, 643.

5. Gallardo H, Bortoluzzi A J and Santos D M, Liq Cryst., 2008, 35, 719.

6. Kolb H C and Sharpless K B, Drug Discov Today, 2003, 8, 1128.

7. Peng wu and Fokin V, Aldrichimica Acta, 2007, 40, 7.

8. Bezborodov V S, Lee C J and Uh K.H, $6^{\text {th }}$ International Conference on Ferroelectric Liquid Crystals; Conference Summaries. Brest, France. 1997, 152.

9. Tome A C, Storr R C and Gilchrist T L, In Science of Synthesis, Ed., Thieme, New York, 2004, 13, 415.

10. Krivopalov V P and Shkurko O P, Russ Chem Rev., 2005, 74, 339.

11. Ferna'ndez O, Dela G, Torre F, Ferna'ndez L, Barbera J and Torres T, Chem Mater., 1997, 9, 3017; (b) Li W R, Su J C, Ke Y C and Lai C K, J Mater Chem., 2001, 1763; (c) Su C, Lee L X, Yu S H, Shih Y K, Su J C, Li F J and Lai C K, Liq Cryst., 2004, 31, 745 .

12. Gilmar Conte, Fernando Ely and Hugo Gallardo, Liq Cryst., 2005, 32, 1213.

13. Hugo Gallardo, Fernando Ely, Bortoluzzi J and Gilmar Conte Liq Cryst., 2005, 32, 667.

14. Cristiano R, Santos O, Conte G and Gallardo H, Liq Cryst., 2006, 33, 997.

15. Gallardo H, Bortoluzzi A J and Santos D M, Liq Cryst., 2008, 35, 719.

16. Perrin D D and Armarego W L F, Prog Polym Sci., 1988, 2, 227.

17. Ian R.Dunkin, Abdunaser A.El Ayeb, Sean L. Gallivan and Michael A. Lynch, $J$ Chem Soc., Perkin Trans, 1997, 2, 1419

18. Lambert Brandsma, Preparative Acetylenic Chemistry, $2^{\text {nd }}$ Ed.; Elsevier, Oxford, $1988,259$. 


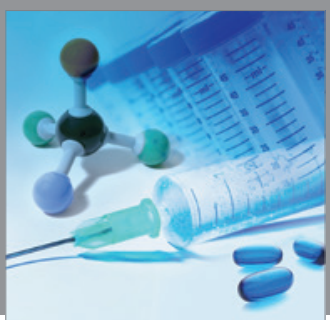

International Journal of

Medicinal Chemistry

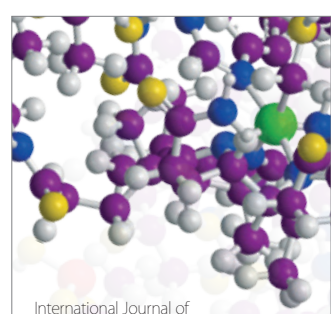

Carbohydrate Chemistry

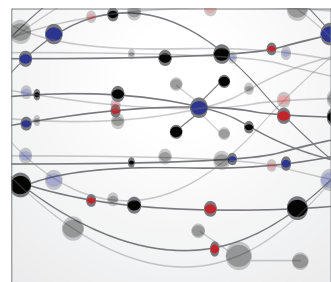

The Scientific World Journal
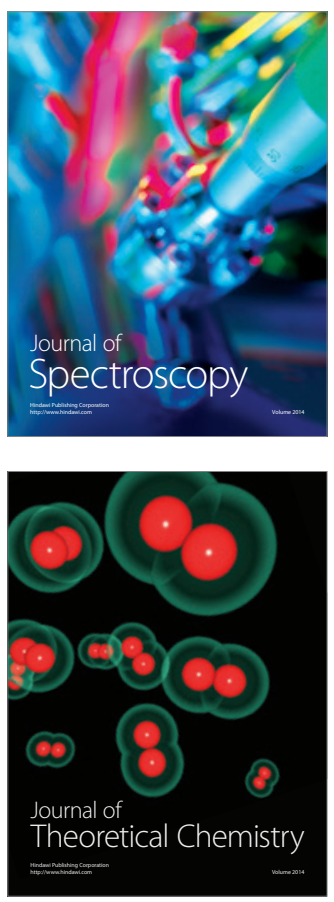
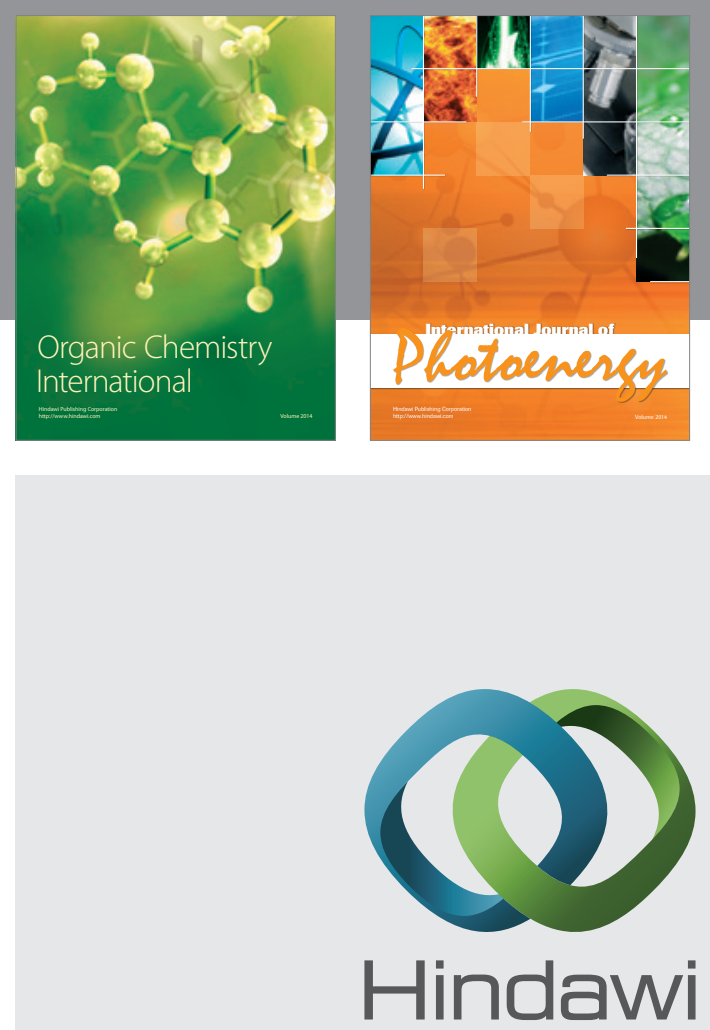

Submit your manuscripts at

http://www.hindawi.com
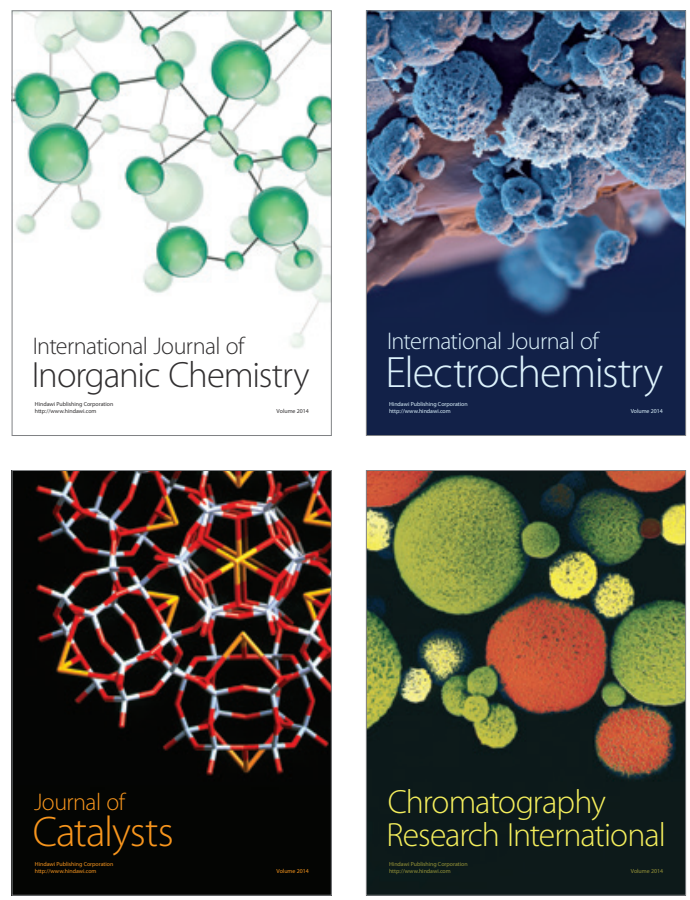
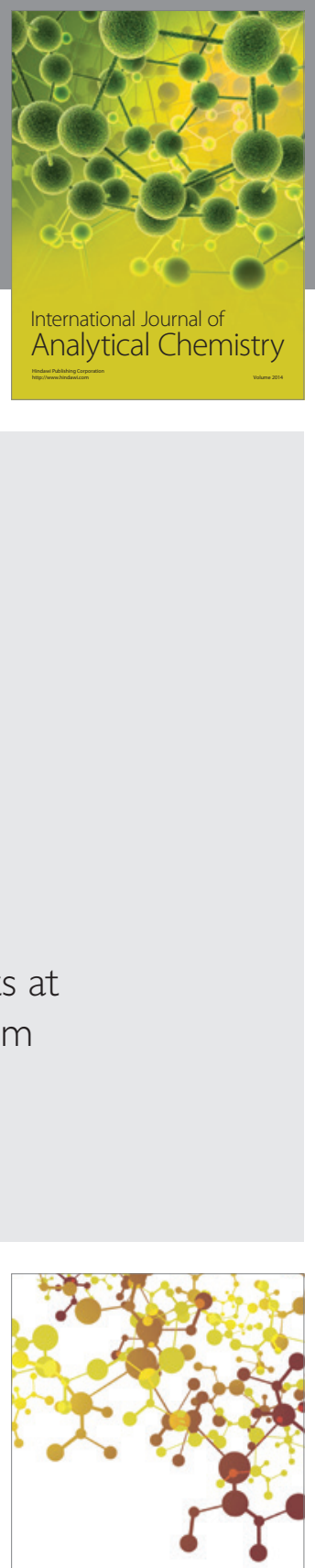

Journal of

Applied Chemistry
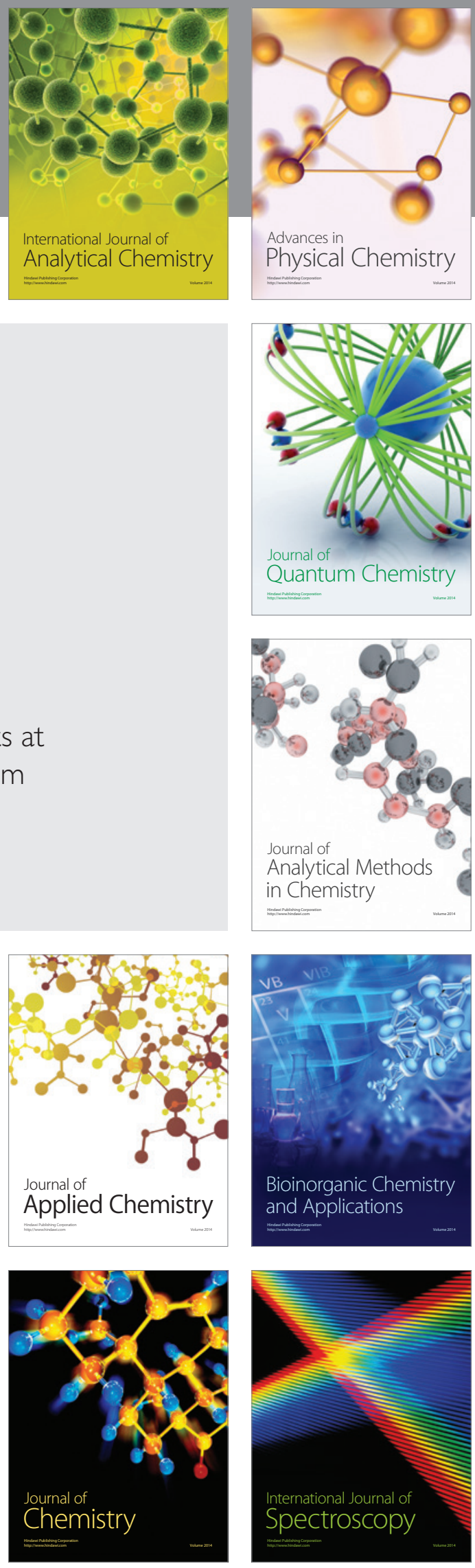